\title{
Electrical Properties of Zn-Phthalocyanine and Poly (3-hexylthiophene) Doped Nematic Liquid Crystal
}

\author{
Y. Karakuş, ${ }^{1}$ M. Okutan, ${ }^{2}$ A. Kösemen, ${ }^{3}$ S. E. San, ${ }^{4}$ Z. Alpaslan, ${ }^{4}$ and A. Demir ${ }^{4}$ \\ ${ }^{1}$ Department of Physics, Sakarya University, Esentepe Campus, 54187 Sakarya, Turkey \\ ${ }^{2}$ Department of Physics, Yildiz Technical University, Davutpasa, 34210 Istanbul, Turkey \\ ${ }^{3}$ Department of Physics, Muş Alparslan University, 49100 Muş, Turkey \\ ${ }^{4}$ Department of Physics, Gebze Institute of Technology, Gebze, 41400 Kocaeli, Turkey \\ Correspondence should be addressed to M. Okutan, mokutan@gmail.com
}

Received 19 April 2011; Revised 24 June 2011; Accepted 10 July 2011

Academic Editor: Gaurav Mago

Copyright (๑) 2011 Y. Karakuş et al. This is an open access article distributed under the Creative Commons Attribution License, which permits unrestricted use, distribution, and reproduction in any medium, provided the original work is properly cited.

An E7 coded nematic liquid crystal was doped with zinc phthalocyanine and poly (3-hexylthiophene). A variety of properties including relaxation time, absorption coefficient, and critical frequency of this doped system were investigated using impedance spectroscopy. The doped systems displayed increased absorption coefficients in the range $0.22-0.55$ and relaxation times from $5.05 \times 10^{-7} \mathrm{~s}$ to $3.59 \times 10^{-6} \mathrm{~s}$ with a decrease in the critical frequency from $3.54 \mathrm{MHz}$ to $2.048 \mathrm{MHz}$.

\section{Introduction}

Liquid crystals (LCs) are molecules that have high nonlinearity and are sensitive to low optical fields. These materials are partially thermally stable in regular phases. Doped LCs have attracted the attention of so many researchers. Because of these characteristics LCs have been used in technological applications intensively $[1,2]$. Poly (3-hexylthiophene) (P3HT) is also a well-known conductive polymer in which holes are dominant charge carriers. This polymer has a high absorption coefficient in almost all along the visible range. Because of this unique property it has been used as an organic-based solar cell $[3,4]$. With the mobility of $0.1 \mathrm{~cm}^{2} / \mathrm{V} \cdot \mathrm{s}$ (which is quite high for a polymer), P3HT has been used in organic field effect transistors as well (OFET) $[5,6]$.

Phthalocyanines $(\mathrm{Pc})$ are also important materials for chemical applications. These thermally stable molecules have high absorption coefficient in the visible range. Pcs which are n-type materials have been used as gas sensors [7], as OFETs [8], and as solar cells [9]. Dielectric properties of Pcs have been investigated in different ways. The dielectric properties of certain $\mathrm{Zn}-\mathrm{Pc}$ molecules are studied by evaporating a thin film of this material on a gold electrode [10]. Dielectric properties of Ni-Pcs are investigated by doping them in a liquid crystal [11]. The complex expression of the dielectric constant can be written as $\varepsilon^{*}=\mathcal{\varepsilon}^{\prime}+i \mathcal{\varepsilon}^{\prime \prime}$ in terms of $\varepsilon^{\prime}$ and $\varepsilon^{\prime \prime}$, which are the real and imaginary parts of dielectric constant, respectively. The frequency-dependent Cole-Cole form of this equation is [12]

$$
\varepsilon^{*}=\varepsilon_{\infty}+\frac{\left(\varepsilon_{s}-\varepsilon_{\infty}\right)}{1+(i \omega \tau)^{1-\alpha}} .
$$

Here $\varepsilon_{s}$ is the value of the low frequency dielectric constant, and $\varepsilon_{\infty}$ is the value of the high frequency dielectric constant in the measured frequency range, $\varpi$ is the angular frequency, $\tau$ is the relaxation time, and $\alpha$ is the absorption coefficient.

To understand the electrical properties of the material with different structures, the dielectric spectroscopy (DS) technique is used. Mentioned real part of the dielectric constant $\varepsilon^{\prime}$ can also be deduced from the following equation:

$$
C=\varepsilon_{o} \varepsilon^{\prime} \frac{A}{d} .
$$

Here $C$ is capacitance, $\varepsilon_{o}$ is the dielectric constant of the free space, $A$ is the surface area, and $d$ is the thickness of the cell. Actually imaginary part of the dielectric constant 


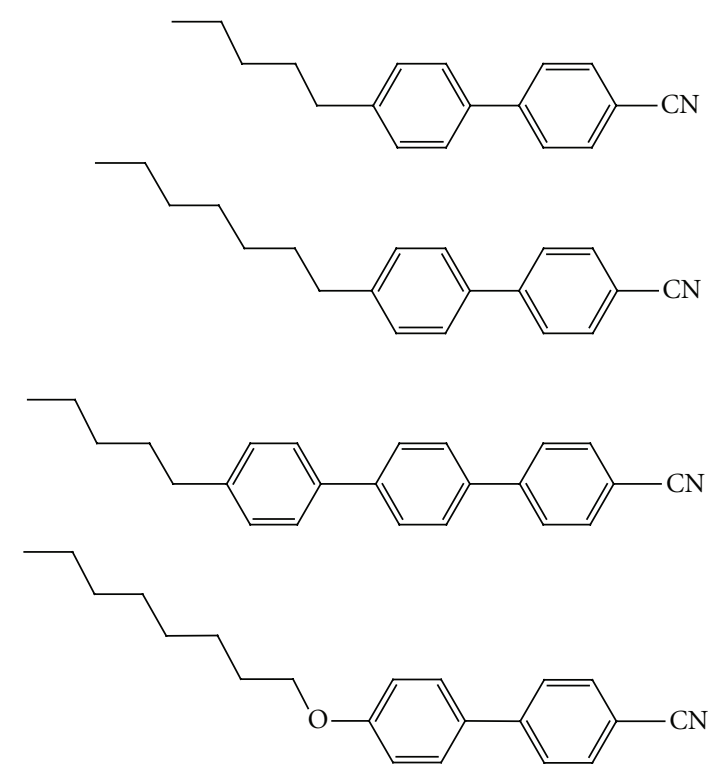

(a)

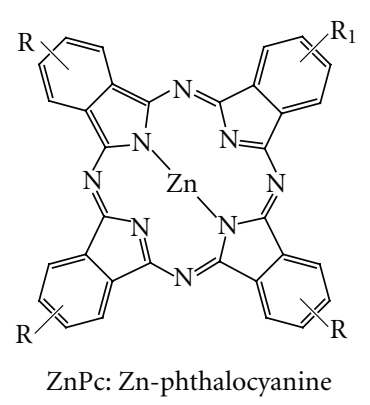

(b)

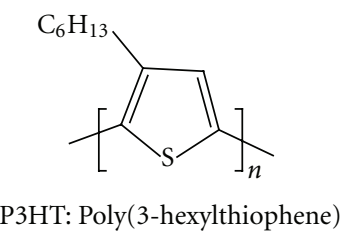

(c)

Figure 1: Chemical structures of: (a) nematic host, E7, (b) Zn-phthalocyanine, (c) Poly(3-hexylthiophene).

$\varepsilon^{\prime \prime}$ is known as the dielectric loss. The dielectric loss $\varepsilon^{\prime \prime}$ is calculated from the following equation:

$$
\varepsilon^{\prime \prime}=\varepsilon^{\prime} \tan \delta,
$$

where $\delta=90-\varphi$ and $\varphi$ is the phase angle. In the scope of this work, relaxation times, absorption coefficient, and critical frequency are measured by DS technique. The ac conductivity and the impedance of these materials, which depend on the frequency, are studied by using the impedance spectroscopy. In fact, impedance spectroscopy is a strong tool which could be applied to overview important parameters of LCs as mentioned in $[13,14]$.

It is also well known that the complex impedance for the LC is expressed as

$$
Z^{*}=Z^{\prime}+Z^{\prime \prime} \text {, }
$$

where $Z^{\prime}$ and $Z^{\prime \prime}$ are the real and the imaginary parts of the complex impedance, respectively, and the real part of the complex impedance is expressed as [15]

$$
Z^{\prime}=R_{s}+\frac{R_{p}}{1+\left(\omega / \omega_{0}\right)^{2}},
$$

where $R_{s}$ is the serial resistance, $\emptyset$ is the angular frequency of the applied field, and $\omega_{0}$ is the characteristic (or natural) angular frequency of the system, $\omega_{0}=1 /\left(R_{\mathrm{p}} C_{\mathrm{p}}\right) . R_{\mathrm{p}}$ is the parallel resistance, and $C_{\mathrm{p}}$ is the parallel capacitance.

\section{Experimental}

The measurement cells are made of two glass slides separated by Mylar sheets having a thickness of about $10 \mu \mathrm{m}$. Before the construction of the cells, Indium tin oxide (ITO) covered glass substrates were spin coated with Polyvinyl alcohol
(PVA) at $2000 \mathrm{rpm}$ and were cured at $50^{\circ} \mathrm{C}$ for 2 hours. The thickness of the coating is about $100 \mathrm{~nm}$. In order to obtain preliminary molecular orientation, these coating layers were exposed to surface treatment of unidirectional rubbing with velvet. This is indeed the so-called planar reorientation, where the LC molecules will be parallel to the ITO walls whatever agent is doped in small amounts. Zn-Pc ( $\mathrm{Zn}$ Phthalocyanine) and P3HT (poly(3-hexylthiophene)) were dissolved in LC under the reinforcement of ultrasonic effect. The structural formula of $\mathrm{Zn}-\mathrm{Pc}, \mathrm{P} 3 \mathrm{HT}$, and the nematic LC (E7) are shown in Figure 1. P3HT was purchased from Sigma-Aldrich Inc. and the Liquid Crystal was attained from Merck.

In this study four different samples were prepared: Undoped E7, a $2 \% \mathrm{w} / \mathrm{w} \mathrm{Zn}-\mathrm{Pc}$, a $2 \% \mathrm{w} / \mathrm{w}$ P3HT, and a mixture of $2 \% \mathrm{w} / \mathrm{w} \mathrm{Zn}-\mathrm{Pc}$ and $2 \% \mathrm{w} / \mathrm{w}$ P3HT, all doped into an E7 host.

Dependency of the impedance on the frequency and the dielectric properties were investigated by making several measurements. Dielectric constant was also calculated from the parallel capacitance technique (see results and discussion). HP 4194A Impedance Analyzer was utilized for these measurements. Complex dielectric response was considered between $1 \mathrm{kHz}$ to $15 \mathrm{MHz}$. RMS amplitude of this device is $\sim 495 \mathrm{mV}$.

\section{Results and Discussion}

Figure 2 shows the real part of the complex impedance for different LC cells. As can be seen in Figure 2, at low frequencies the real part of the impedance for Pc-Zn and P3HT doped LC samples indicates a plateau region. After the plateau region, the impedance decreases drastically with the increasing frequency. At high frequencies, the impedance is 


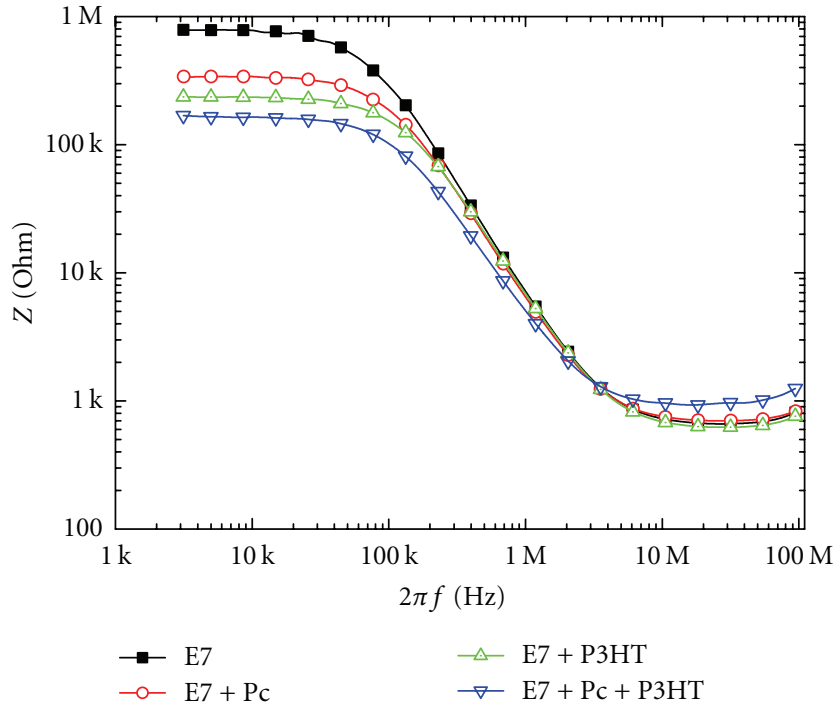

FIGURE 2: Variation of the impedance with the frequency.

stable with the increasing frequency. Compared to those of $\mathrm{Zn}-\mathrm{Pc}$ and P3HT doped samples, the impedance values of $\mathrm{Zn}-\mathrm{Pc}+\mathrm{P} 3 \mathrm{HT}$ doped LC samples are higher.

In the LC dielectric materials, $\varepsilon^{\prime}$ decreases as the frequency increases. This decrease is mainly due to the polarisability of the molecules, ionic conductivity, and interfacial orientation. Figure 3 shows this decrease of $\varepsilon^{\prime}$ with the frequency. The $\varepsilon^{\prime}$ initially decreases rapidly with the increase in the frequency, and as the frequency increases $\varepsilon^{\prime}$ attains a constant value.

The dielectric strength is the difference between the dielectric values at minimum and at maximum frequencies. In the impedance spectroscopy technique the dielectric strength $\Delta \varepsilon$ is expressed as

$$
\Delta \varepsilon=\varepsilon_{s}-\varepsilon_{\infty},
$$

where $\varepsilon_{s}$ and $\varepsilon_{\infty}$ are the minimum and maximum components of the dielectric constant. Figure 3 shows clearly that the doping of $\mathrm{Zn}-\mathrm{Pc}$ increases the dielectric strength of the sample and that the doping of P3HT increases it further. In fact, values of the dielectric strength $\Delta \varepsilon$ deduced from Figure 3 are given in Table 1. With the doping, $\Delta \varepsilon$ values increased from 6.75 to 10.29 . This shows that when the sample is doped with the mixture of both these doping materials, then dielectric strength increased further. Overall uncertainty in dielectric measurements was 0.02 at $(k=2)$ [16].

If the dielectric medium in the measurement cell is subject to an electric field, dielectric constant is supposed to decrease as shown in (1) [12]. Also one can say that $\varepsilon_{\infty}$ value is tending to zero at high frequencies while $\varepsilon_{s}$ can take various values at low frequencies for different LC samples.

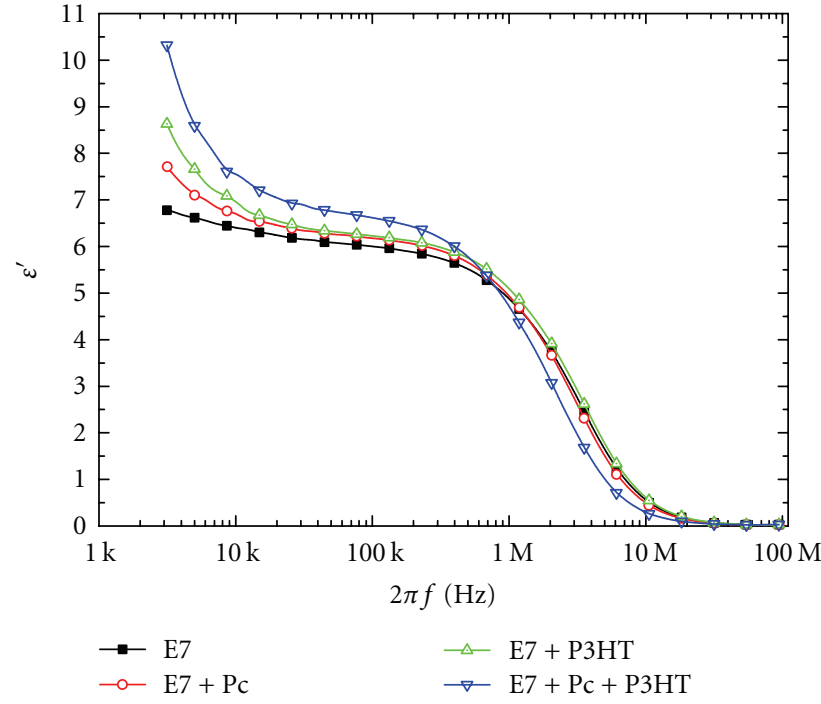

FIgure 3: Dependency of the real dielectric constant on the frequency.

When the real part of the dielectric constant in (1) is written in the Cole-Cole equation form [12] it becomes

$\varepsilon^{\prime}(\varpi)=\varepsilon_{\infty}+\left(\varepsilon_{o}-\varepsilon_{\infty}\right) \frac{1+(\varrho \tau)^{1-\alpha} \sin (1 / 2) \alpha \pi}{1+2(\omega \tau)^{1-\alpha} \sin (1 / 2) \alpha \pi+(\omega \tau)^{2(1-\alpha)}}$,

where $\tau$ is the relaxation time, and $\alpha$ is the absorption coefficient. The change of the dielectric constant in Figure 3 is extrapolated according to (7). Values of $\tau$ and $\alpha$ are calculated from this extrapolation and are shown in Table 1. While the value of the relaxation time of undoped E7 is about $5.08 \times$ $10^{-7} \mathrm{~s}$, in $\mathrm{Zn}-\mathrm{Pc}$ doped E7, it becomes $8.29 \times 10^{-7} \mathrm{~s}$, and in P3HT doped E7 it reaches the value of $1.41 \times 10^{-6} \mathrm{~s}$. When both of these materials are doped into E7, the relaxation time increases again and reaches the value of $3.59 \times 10^{-6} \mathrm{~s}$. The absorption coefficient $\alpha$ is also increased with doping (See Table 1).

Imaginary dielectric constant is indeed the measure of the dissipation factor $(\tan (\delta))$. In Figure 4, it is observed that the doping increases these dissipation factors at low frequencies. A decrease in the value of the critical frequency is also observed. The critical frequencies, which correspond to the maximum imaginary part of the dielectric constant, were acquired from Figure 4 and are shown in Table 1. While the value of the critical frequency for undoped E7 is $3.54 \mathrm{MHz}$, it becomes $3.26 \mathrm{MHz}$ in $\mathrm{Zn}-\mathrm{Pc}$ doped E7, it decreases to $2.88 \mathrm{MHz}$ in P3HT doped E7, and it decreases further to 2.04 MHz when E7 is doped with $\mathrm{Zn}-\mathrm{Pc}+\mathrm{P} 3 \mathrm{HT}$.

Dissipation factor is the rate of the real impedance over the imaginary one. When the values of the real and imaginary impedance are close, the dissipation factor becomes smaller. This change in the dissipation factor can be seen in Figure 5 . Decrease in the critical frequency with doping could be observed in Figure 5 as well. At low frequencies, dissipation factor is small, but it gets higher at high frequencies with the increasing real impedance. 
Table 1: The dielectric strength $\Delta \varepsilon$, the relaxation time $\tau$, the absorption coefficient $\alpha$, and the critical frequency $f_{c}$ of the samples.

\begin{tabular}{lcccc}
\hline Sample & $\Delta \varepsilon$ & $\tau(s)$ & $\alpha$ & $f_{\mathrm{c}}(\mathrm{Hz})$ \\
\hline E7 & 6.75 & $5.08 \times 10^{-7}$ & 0.22 & $3.54 \times 10^{6}$ \\
E7 + Zn-Pc & 7.68 & $8.26 \times 10^{-7}$ & 0.37 & $3.2 \times 10^{6}$ \\
E7 + P3HT & 8.6 & $1.41 \times 10^{-6}$ & 0.51 & $2.88 \times 10^{6}$ \\
E7 + ZnPc + P3HT & 10.29 & $3.59 \times 10^{-6}$ & 0.55 & $2.04 \times 10^{6}$ \\
\hline
\end{tabular}

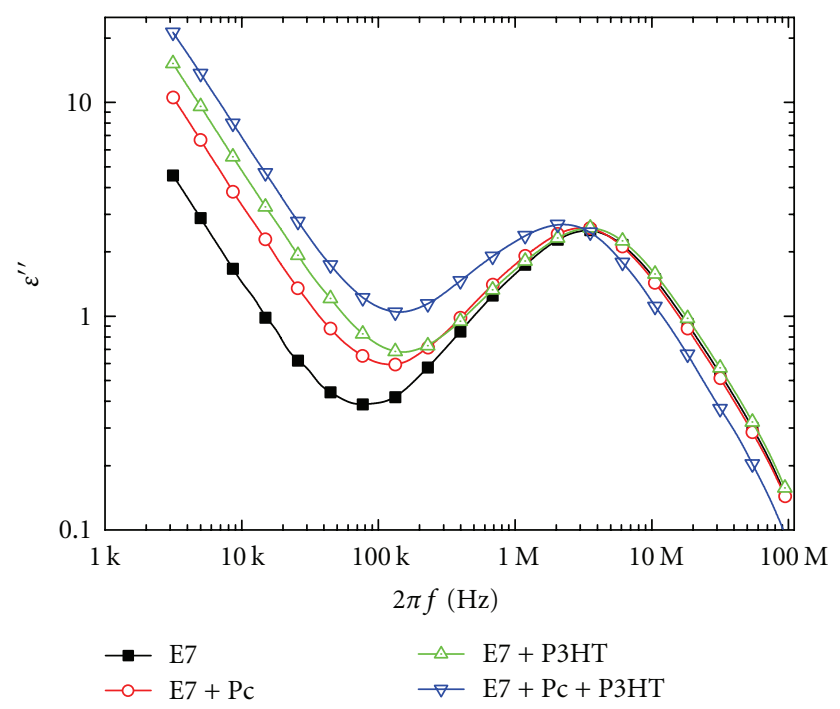

Figure 4: Dependency of the imaginary dielectric constant on the frequency.

TABle 2: The dielectric constants $\varepsilon_{o}$, $\varepsilon_{\infty}$, and $\varepsilon_{\max }$ of the samples.

\begin{tabular}{lccc}
\hline Specimens & $\varepsilon_{o}$ & $\varepsilon_{\infty}$ & $\varepsilon_{\max }$ \\
\hline E7 & 67.81 & 0.03 & 2.52 \\
E7 + Pc & 77.09 & 0.03 & 2.62 \\
E7 + P3HT & 86.33 & 0.03 & 2.62 \\
E7 + Pc + P3HT & 10.32 & 0.03 & 2.72 \\
\hline
\end{tabular}

When the value of absorption coefficient $(\alpha)$ is zero, the material shows Debye type relaxation, if the values of $\alpha$ gets bigger than zero, and less than one, the material shows non-Debye type relaxation [12]. Existence of Debye type relaxation can be determined from the Cole-Cole plots. As shown in Figure 6, the centers of the arcs are located below the $x$-axis for all samples, indicating that non-Debye type relaxation is dominant when there is no bias field applied $[15,16]$. From the analysis of the Cole-Cole plots that are shown in Figure 6, one can get information about the dielectric constants at minimum and maximum frequencies.

The value of the dielectric constant at the maximum point of the semicircles $\varepsilon_{\max }$ can also be obtained from these plots for the different doping materials. All the dielectric constants deduced from Cole-Cole plots are shown in Table 2.

Cole-Cole plots can give information about the equivalent circuit structures. Our LC cells show properties of a parallel RC equivalent circuit in series to a resistance.

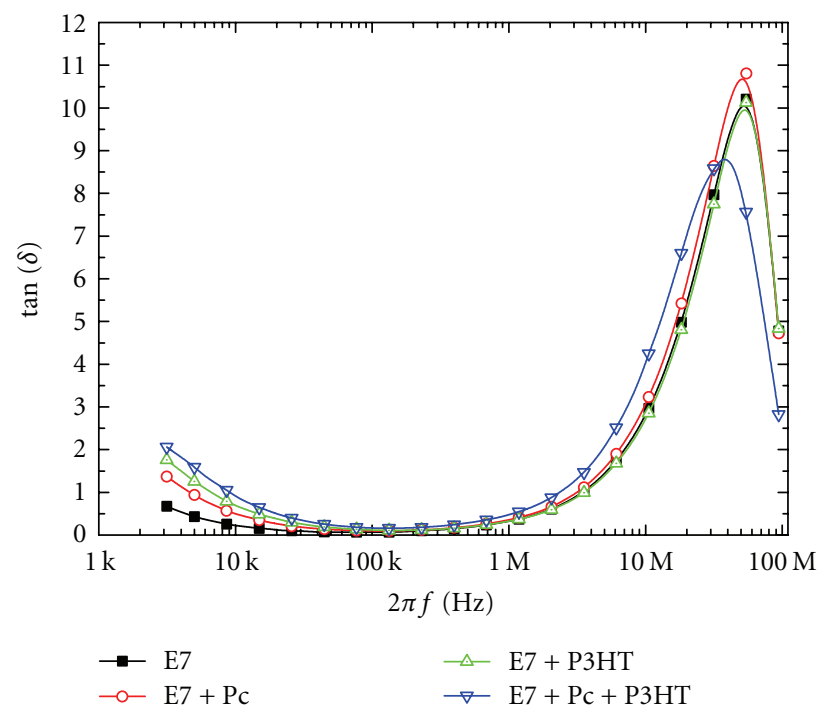

FIGURE 5: Variation of the dissipation factor with the frequency.

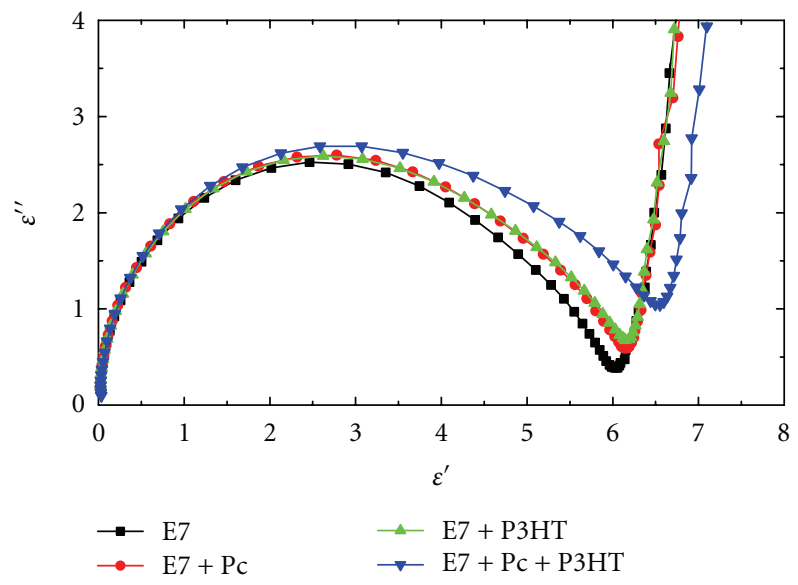

Figure 6: Cole-Cole plots of the pure and the doped samples.

Critical frequency decreases exponentially with the absorption coefficient $\alpha$ with the addition of different doping materials (Figure $7(\mathrm{a})$ ), and the relaxation time increases exponentially with the increase of the absorption coefficient $\alpha$ (Figure 7(b)).

\section{Conclusions}

In this study, the doping of Zn-Pc and P3HT into E7 decreased the impedance. From this study we concluded that P3HT is a better conductor than $\mathrm{Zn}-\mathrm{Pc}$, and when the sample is doped with both of these materials in the same proportion, then the impedance is decreased further. A study of the critical frequency, relaxation time, and absorption coefficients using DS is also carried out. As a result, we have noticed that the doping decreased the critical frequency and increased the conductivity. It also increased the relaxation time. Increase in the values of absorption coefficient by doping implies the non-Debye type relaxation behaviors in 


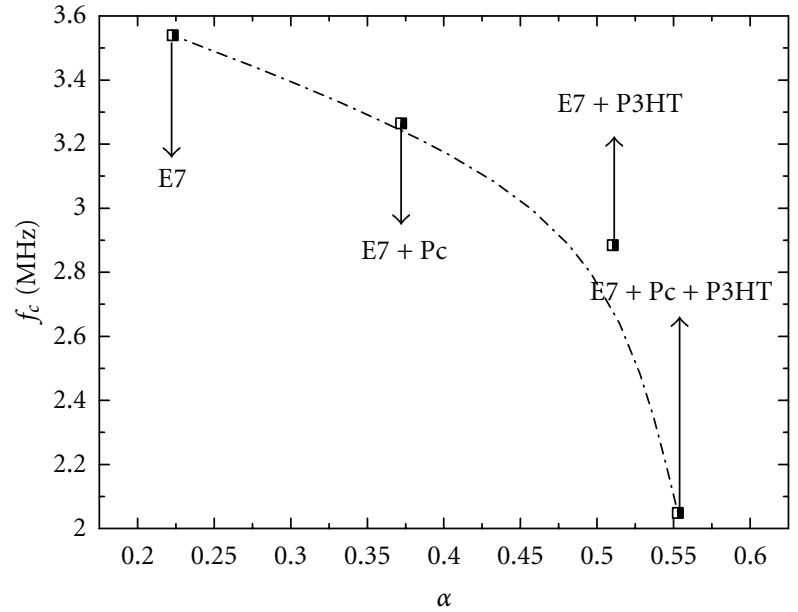

(a)

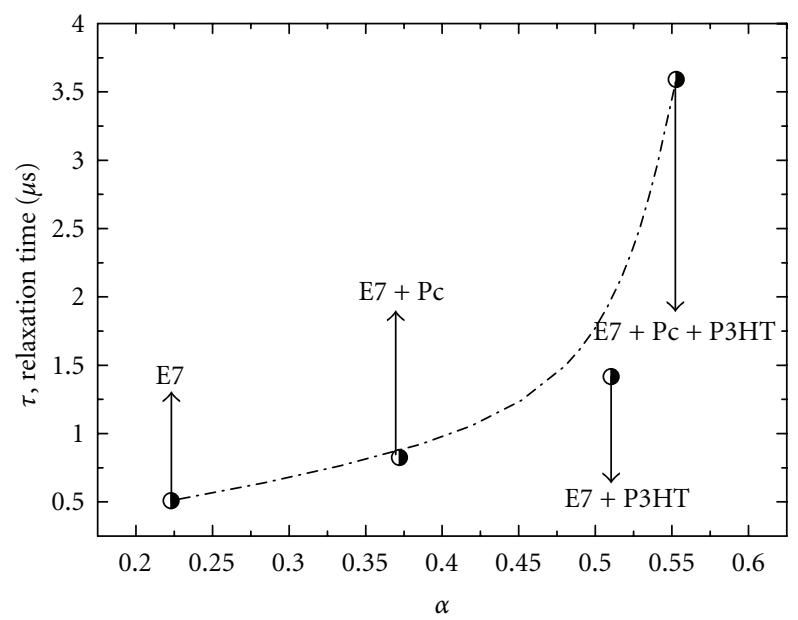

(b)

Figure 7: (a) Variation of the critical frequency with $\alpha$. (b) Variation of the relaxation time with $\alpha$.

the proposed LC sample, which is a novel configuration caused by the doping of P3HT and Zn-Pc.

\section{Acknowledgment}

Special thanks are due to Dr. Burak Esat from Fatih University for providing the authors with $\mathrm{Zn}$-phthalocyanine.

\section{References}

[1] F. Simoni and O. Francescangeli, "Effects of light on molecular orientation of liquid crystals," Journal of Physics Condensed Matter, vol. 11, no. 41, p. R439, 1999.

[2] Y. J. Wang and G. O. Carlisle, "Optical properties of dispersered-1-doped nematic liquid crystal," Journal of Materials Science: Materials in Electronics, vol. 13, no. 3, pp. 173-178, 2002.

[3] Y. Y. Kim, S. Cook, S. M. Tuladhar et al., "A strong regioregularity effect in self-organizing conjugated polymer films and high-efficiency polythiophene: fullerene solar cells," Nature Materials, vol. 5, no. 3, pp. 197-203, 2006.

[4] M. C. Scharber, D. Mühlbacher, M. Koppe et al., "Design rules for donors in bulk-heterojunction solar cells-towards $10 \%$ energy-conversion efficiency," Advanced Materials, vol. 18, no. 6, pp. 789-794, 2006.

[5] D. H. Kim, Y. D. Park, Y. S. Jang et al., "Enhancement of fieldeffect mobility due to surface-mediated molecular ordering in regioregular polythiophene thin film transistors," Advanced Functional Materials, vol. 15, no. 1, pp. 77-82, 2005.

[6] Z. Bao, A. Dodabalapur, and A. J. Lovinger, "Soluble and processable regioregular poly (3-hexylthiophene) for thin film field-effect transistor applications with high mobility," Applied Physics Letters, vol. 69, no. 26, pp. 4108-4110, 1996.

[7] R. Zhou, F. Josse, W. Göpel, Z. Z. Özturk, and Ö. Bekaroglu, "Phthalocyanines as sensitive materials for chemical sensors," Applied Organometallic Chemistry, vol. 10, no. 8, pp. 557-577, 1996.

[8] T. B. Singh, R. Koeppe, N. S. Sariciftci, M. Morana, and C. J. Brabec, "Monitoring the channel formation in organic fieldeffect transistors via photoinduced charge transfer," Advanced Functional Materials, vol. 19, no. 5, pp. 789-795, 2009.

[9] C. J. Brabec, N. S. Sariciftci, and J. C. Hummelen, "Plastic solar cells," Advanced Funtional Materials, vol. 11, no. 1, pp. 15-26, 2001.

[10] H. M. Zeyada and M. M. El-Nahass, "Electrical properties and dielectric relaxation of thermally evaporated zinc phthalocyanine thin films," Applied Surface Science, vol. 254, no. 6, pp. 1852-1858, 2008.

[11] O. Köysal, M. Okutan, M. Durmuş, F. Yakuphanoglu, S. E. San, and V. Ahsen, "Diffraction efficiency and dielectric relaxation properties of nickel phthalocyanine doped nematic liquid crystal," Synthetic Metals, vol. 156, no. 1, pp. 58-64, 2006.

[12] G. G. Raju, Dielectrics in Electric Fields, Marcel Dekker, New York, NY, USA, 2003.

[13] S. E. San, M. Okutan, O. Köysal, and Y. Yerli, "Carbon nanoparticles in nematic liquid crystals," Chinese Physics Letters, vol. 25, no. 1, pp. 212-215, 2008.

[14] M. Okutan, F. Yakuphanoglu, S. E. San, and O. Köysal, "Impedance spectroscopy and dielectric anisotropy-type analysis in dye-doped nematic liquid crystals having different preliminary orientations," Physica B, vol. 368, no. 1-4, pp. 308-317, 2005.

[15] A. Popova, S. Raicheva, E. Sokolova, and M. Christov, "Frequency dispersion of the interfacial impedance at mild steel corrosion in acid media in the presence of benzimidazole derivatives," Langmuir, vol. 12, no. 8, pp. 2083-2089, 1996.

[16] ISO, Guide to the Expression of Uncertainty of Measurement, 1st edition, 1993. 

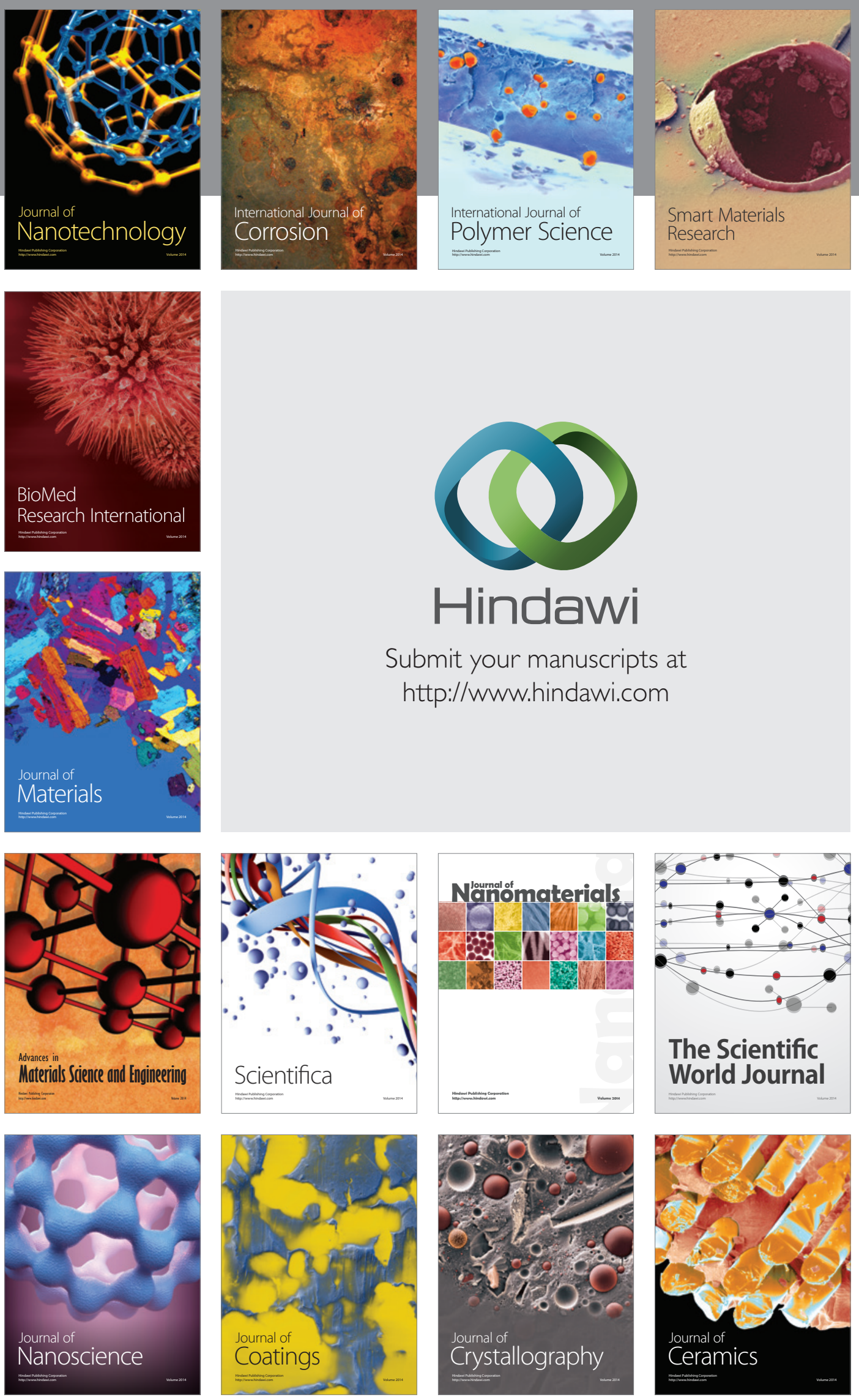

The Scientific World Journal

Submit your manuscripts at

http://www.hindawi.com

\section{World Journal}

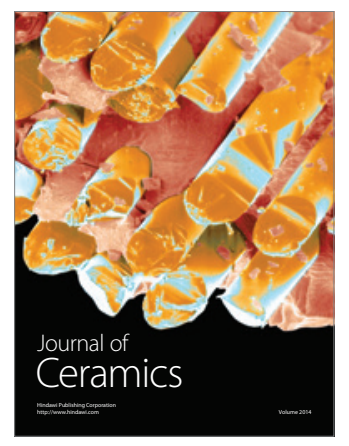

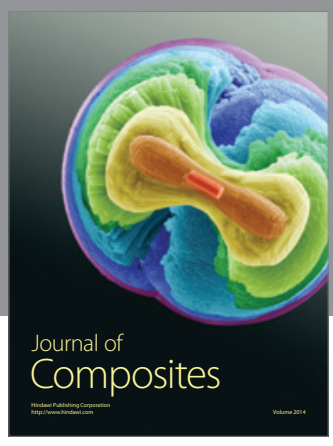
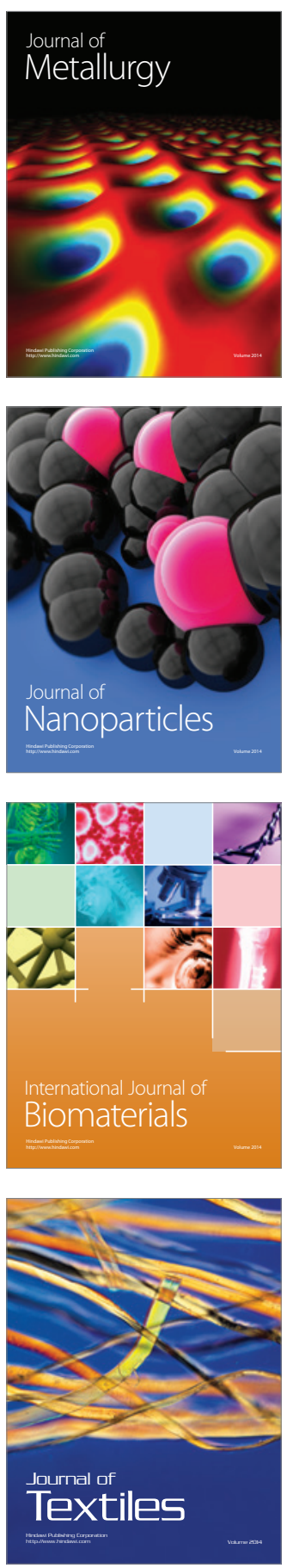